\title{
Un acercamiento coinductivo al análisis real
}

\author{
Guillermo Ortiz Rico*, Lina Isabel Triviño Viera \\ Universidad del Valle, Departamento de Matemática, Cali, Colombia.
}

Resumen. La coinducción, un concepto dual a la inducción, ha sido descubierto y estudiado recientemente. Una forma sencilla de entender su naturalidad es observando que este se refiere a los puntos fijos más grandes, mientras que la inducción se refiere a los más pequeños. Inicialmente el soporte técnico de la coinducción estaba en la teoría de retículos a través de los puntos fijos más grandes, ahora dicho soporte se centra en el lenguaje de categorías a través de las $F$-coálgebras finales. Las $F$-coálgebras son un concepto dual a la generalización de $F$-álgebras para un funtor $F$. En el presente trabajo nos centraremos en un tipo muy particular de $F$-coálgebras: los autómatas de cadena. Nuestro objetivo será usar el marco de los autómatas de cadena para ilustrar el carácter coinductivo del análisis real a través de resultados clásicos como el teorema fundamental del cálculo, las series de Taylor y la solución de ciertas ecuaciones diferenciales.

Palabras clave: Categorias, Álgebra, Coálgebra, Inducción, Coinducción. MSC2010: 18A05, 18C10, 03B70, 68Q65.

\section{A coinductive approach to real analysis}

\begin{abstract}
Coinduction is a dual concept to induction; it has been discovered and studied recently. A simple way to understand its naturalness is noting that it refers to the largest fixed points, while induction refers to smallest fixed points. Originally the technical support of the coinduction was the lattices theory through the largest fixed points; now this support is in the language categories through the final $F$-coalgebras. The $F$-coalgebras is a dual concept of generalization to algebras for a functor $F$. In this paper we focus on a particular type of $F$-coalgebras: stream automata. Our aim will be to use the framework of stream automata to illustrate the coinductive character of real analysis through classical results as the fundamental theorem of calculus, Taylor series and the solution of certain differential equations.
\end{abstract}

Keywords: Categories, Algebra, Coalgebra, Induction, Coinduction.

\footnotetext{
${ }^{*} E$-mail: guillermo.ortiz@correounivalle.edu.co Recibido: 14 de octubre de 2016, Aceptado: 20 de junio de 2017.

Para citar este artículo: G. Ortiz Rico, L.I Triviño Viera, Un acercamiento coinductivo al análisis real, Rev. Integr. Temas Mat. 35 (2017), No. 1, 103-125.
} 


\section{Introducción}

La coinducción ${ }^{1}$, un concepto dual a la inducción, descubierto y estudiado recientemente, está en desarrollo progresivo: más y más áreas de aplicación han surgido con resultados contundentes. En términos de la teoría de retículos, la inducción se refiere los puntos fijos más pequeños, y la coinducción se refiere a los más grandes. Los puntos fijos más grandes son tan naturales como los puntos fijos más pequeños; así pues, la coinducción es tan natural como la inducción. En desarrollos recientes el lenguaje de categorías permite formalizaciones más amplias, fijando un funtor $F$ para la inducción a través de las $F$ álgebras iniciales, y dualmente para la coinducción con las $F$-coálgebras finales. En este contexto, centrándonos en los autómatas de cadena como $F$-coálgebras esperamos ilustrar el carácter coinductivo del análisis real. En la búsqueda de este objetivo presentamos cuatro secciones. En la primera sección presentamos, casi a manera de introducción, un acercamiento al desarrollo de la generalización del concepto de álgebra en la denominada Álgebra Universal, hasta llegar a la definición de $F$-álgebras; ilustraremos, con el Principio de Inducción Matemática en las álgebras tipo 1,0, que al considerar la categoría $A \lg _{\mathbf{F}}$ de todas las $F$-álgebras, si esta categoría tiene objeto inicial se tiene un Principio de Inducción. En la segunda sección formalizamos la definición de las $F$-coálgebras, un concepto dual a las $F$-álgebras; al considerar la categoría Coalg $_{\mathbf{F}}$ de todas las $F$ coálgebras, si esta categoría tiene objeto final se tiene un Principio de Coinducción; aquí se muestran como ejemplos las Máquinas de Mealy, los Modelos de Kripke y un ejemplo de coálgebra final. En la tercera sección nos centraremos en los autómatas de cadena; se estudia el comportamiento equivalente como bisimulación; se muestran algunos resultados del cálculo; y a partir de la estructura de $\mathbb{R}$ se "construye" una estructura algebraica en $\mathbb{R}^{\omega}$. En la cuarta sección se mostrará la existencia y unicidad de la solución de algunas ecuaciones diferenciales en cadenas empleando la coinducción y la finalidad de $\mathbb{R}^{\omega}$ en la categoría de los autómatas, ilustrando con ejemplos particulares la solución de ecuaciones diferenciales y sistemas de ecuaciones diferenciales $2 \times 2$.

\section{2. Álgebras e Inducción}

A pesar de la extensa historia del desarrollo del álgebra abstracta podemos ubicar momentos muy importantes. El primero; el desarrollo del álgebra simbólica durante el siglo XIX en Gran Bretaña que trata el álgebra como combinaciones de signos y símbolos por medio de leyes arbitrarias convirtiéndose la aritmética en una ciencia más general; estos desarrollos fueron liderados por Peacock, de Morgan y Boole. El segundo, a inicios del siglo XX, los trabajos de Emmy Noether y Emil Artin, cuyas ideas se concretan en el texto de Álgebra de van der Waerden de 1930, que determina un cambio en la imagen de los conceptos algebraicos. En este texto se estudian grupos, anillos y cuerpos como aparecen en la mayor parte de los textos de álgebra abstracta ó moderna del siglo XX. El tercero; en las cercanías de 1950: antes de esta fecha las conexiones entre el álgebra universal y la teoría de retículos por Birkhoff y Ore; y después de la misma fecha, las del álgebra universal y la teoría de modelos por Tarski y Malcev. El cuarto; en las cercanías de 1970: las conexiones entre el álgebra universal y la teoría de categorías por Lawvere y Benabou, entre otros. Presentamos algunos elementos básicos del álgebra universal, que

\footnotetext{
${ }^{1}$ Agradecemos a los jurados anónimos por sus valiosas observaciones, que nos han permitido la consolidación de este panorama introductorio en coálgebras y coinducción.
} 
se pueden ampliar en [2], que junto a algunos ejemplos simples ilustran un acercamiento a los primeros tres momentos antes comentados.

Para $A$ un conjunto no vacío $A^{0}=\{\emptyset\}$, y $A^{n}=\left\{\left(a_{1}, \ldots, a_{n}\right): a_{i} \in A, 1 \leq i \leq n\right\}$ para $n$ número natural positivo, una operación n-aria en $A$ es una función $f: A^{n} \rightarrow A$. Decimos que $n$ es la aridad de $f$ y escribimos $a(f)=n$. Para el caso $n=0$ la operación $f$ se dice nularia y está completamente determinada por la imagen $f(\emptyset)$ en $A$, es decir, se trata de la selección de un elemento de $A$. Además es usual decir que una operación $f$ en $A$ es unaria, binaria o ternaria si su aridad es uno, dos o tres, respectivamente.

Para establecer las álgebras del mismo tipo, fijamos un lenguaje de álgebras como un conjunto $\Sigma$ de símbolos de función, tal que cada $f \in \Sigma$ tiene asociado un número natural $a(f)$, la aridad de $f$. Entonces $\mathbf{A}$ es una $\Sigma$-álgebra o un álgebra tipo $\Sigma$ siempre que $\mathbf{A}=\left\langle A, \Sigma^{\mathbf{A}}\right\rangle$ donde $A$ es un conjunto no vacío y $\Sigma^{\mathbf{A}}$ es una familia de operaciones finitarias en $A$ indexadas por el lenguaje $\Sigma$, es decir, hay una correspondencia de cada símbolo de función $n$-ario $f \in \Sigma$ con una operación $n$-aria $f^{\mathbf{A}} \in \Sigma^{\mathbf{A}}$. El conjunto $A$ es llamado el universo de $\mathbf{A}=\left\langle A, \Sigma^{\mathbf{A}}\right\rangle$ y las $f^{\mathbf{A}}$ son llamadas las operaciones fundamentales de A. Si $\Sigma$ es finito, $\Sigma=\left\{f_{1}, \ldots, f_{k}\right\}$, escribimos $\mathbf{A}=\left\langle A, f_{1}^{\mathbf{A}}, \ldots, f_{k}^{\mathbf{A}}\right\rangle$ para $\left\langle A, \Sigma^{\mathbf{A}}\right\rangle$, y es usual adoptar la siguiente convención: $a\left(f_{1}\right) \geq \ldots \geq a\left(f_{k}\right)$.

Ejemplo 2.1. Ejemplos de álgebras:

- Un grupo es un álgebra $\mathbf{G}=\left\langle G, \cdot,^{-1}, e\right\rangle$ con $a(\cdot)=2, a\left({ }^{-1}\right)=1$ y $a(e)=0$, en el cual se satisfacen las siguientes identidades:

$\mathbf{G}_{\mathbf{1}}: x \cdot(y \cdot z)=(x \cdot y) \cdot z$,

$\mathbf{G}_{\mathbf{2}}: x \cdot e=e \cdot x$,

$\mathbf{G}_{\mathbf{3}}: x \cdot x^{-1}=x^{-1} \cdot x=e ;$

$\mathbf{G}$ es abeliano si satisface la condición adicional

$\mathbf{G}_{\mathbf{4}}: x \cdot y=y \cdot x$.

- Un anillo es un algebra $\mathbf{R}=\langle R,+, \cdot,-, 0\rangle$, un lenguaje de algebras con $a(+)=2$, $a(\cdot)=2, a(-)=1$ y $a(0)=0$, en el cual tenemos:

$\mathbf{R}_{\mathbf{1}}:\langle R,+,-, 0\rangle$ es un grupo abeliano,

$\mathbf{R}_{\mathbf{2}}: x \cdot(y \cdot z)=(x \cdot y) \cdot z$,

$\mathbf{R}_{3}: x \cdot(y+z)=x \cdot y+x \cdot z \mathrm{y}(x+y) \cdot z=x \cdot z+y \cdot z$.

- Un retículo es un álgebra $\mathbf{L}=\langle L, \vee, \wedge\rangle$ con $a(\vee)=a(\wedge)=2$, las cuales satisfacen:

L1: a) $x \vee y=y \vee x$

b) $x \wedge y=y \wedge x$

(Ley conmutativa)

L2: a) $(x \vee y) \vee z=x \vee(y \vee z)$,

b) $(x \wedge y) \wedge z=x \wedge(y \wedge z)$

(Ley Asociativa)

L3: a) $x \vee x=x$,

b) $x \wedge x=x$

(Ley de Idempotencia)

Vol. 35, No. 1, 2017] 
L4: a) $x=x \vee(x \wedge y)$,

b) $x=x \wedge(x \vee y)$.

(Ley de Absorción)

Decimos que los grupos son álgebras tipo 2,1,0; los anillos (unitarios) 2, 2,1,0,0 y los retículos son 2,2. En el contexto del álgebra universal se presentan los desarrollos propios como subálgebras y homomorfismo. Por ahora, sólo para ilustrar la elegancia de esta presentación, introducimos estos últimos. Si A y $\mathbf{B}$ son dos $\Sigma$-álgebras, una función $\alpha: \mathbf{A} \rightarrow \mathbf{B}$ es un homomorfismo si, para toda operación $n$-aria $f \in \Sigma$ y para todos $a_{1}, \ldots, a_{n} \in A$, tenemos $\alpha f^{\mathbf{A}}\left(a_{1}, \ldots, a_{n}\right)=f^{\mathbf{B}}\left(\alpha a_{1}, \ldots, \alpha a_{n}\right)$.

Ahora suponemos en nuestro lector los conocimientos básicos de la Teoría de Categorías, para así dar un acercamiento al cuarto momento de la introducción. Supongamos que estamos en la categoría de los conjuntos Set y tenemos un tipo de lenguaje fijo $\Sigma=$ $\left\{f^{(2)}, g^{(2)}, h^{(1)}, k^{(0)}\right\}$; entonces una $\Sigma$-álgebra está dada por $\mathbf{A}=\left\langle A, \Sigma^{\mathbf{A}}\right\rangle$, donde $\Sigma^{\mathbf{A}}=$ $\left\{f^{\mathbf{A}}, g^{\mathbf{A}}, h^{\mathbf{A}}, k^{\mathbf{A}}\right\} \quad$ y $\quad f^{\mathbf{A}}: A \times A \rightarrow A, \quad g^{\mathbf{A}}: A \times A \rightarrow A, \quad h^{\mathbf{A}}: A \rightarrow A$ y $k^{\mathbf{A}}: 1 \rightarrow A$ (donde $1=\{\emptyset\}$ ).

Consideremos el morfismo ${ }^{2} \alpha=f^{\mathbf{A}}+g^{\mathbf{A}}+h^{\mathbf{A}}+k^{\mathbf{A}}:(A \times A)+(A \times A)+A+1 \rightarrow A$, y veamos que este morfismo representa los morfismos $f^{\mathbf{A}}, g^{\mathbf{A}}, h^{\mathbf{A}}$ y $k^{\mathbf{A}}$ :

- El morfismo $f^{\mathbf{A}}=A \times A \stackrel{i}{\longrightarrow}(A \times A)+(A \times A)+A+1 \stackrel{\alpha}{\longrightarrow} A$, donde el primer morfismo es la inclusión de $A \times A$ en la primera aparición del producto $A \times A$ en $(A \times A)+(A \times A)+A+1$.

- El morfismo $g^{\mathbf{A}}=A \times A \stackrel{i}{\longrightarrow}(A \times A)+(A \times A)+A+1 \stackrel{\alpha}{\longrightarrow} A$, donde el primer morfismo es la inclusión de $A \times A$ en la segunda aparición de $A \times A$ en $(A \times A)+$ $(A \times A)+A+1$.

- El morfismo $h^{\mathbf{A}}=A \stackrel{i}{\longrightarrow}(A \times A)+(A \times A)+A+1 \stackrel{\alpha}{\longrightarrow} A$, donde el primer morfismo es la inclusión de $A$ en $(A \times A)+(A \times A)+A+1$.

- El morfismo $k^{\mathbf{A}}=1 \stackrel{i}{\longrightarrow}(A \times A)+(A \times A)+A+1 \stackrel{\alpha}{\longrightarrow} A$, donde el primer morfismo es la inclusión de 1 en $(A \times A)+(A \times A)+A+1$.

Entonces podemos pensar un álgebra tipo $2,2,1,0$ por $\mathbf{A}=\langle A, \alpha\rangle$. Si además consideramos el funtor $F:$ Set $\rightarrow$ Set definido para los objetos por $F(A)=(A \times A)+(A \times A)+A+1$, tenemos que $\alpha: F(A) \rightarrow A$. En un sentido bastante preciso observamos que el tipo de álgebra 2,2,1,0 es justamente guardado por la definición del funtor $F$ en los objetos.

De otro lado, si $\phi: A \rightarrow B$ y tenemos dos álgebras de este mismo tipo $(2,2,1,0),\langle A, \alpha\rangle$ y $\langle B, \beta\rangle$ donde $\alpha=f^{\mathbf{A}}+g^{\mathbf{A}}+h^{\mathbf{A}}+k^{\mathbf{A}} \quad$ y $\beta=f^{\mathbf{B}}+g^{\mathbf{B}}+h^{\mathbf{B}}+k^{\mathbf{B}}$, definimos $F$ sobre los morfismos por $F(\phi)=(\phi \times \phi)+(\phi \times \phi)+(\phi)+1$. La condición de que $\phi$ es un homomorfismo se reduce a que el siguiente diagrama conmuta:

\footnotetext{
${ }^{2}$ En la literatura es usual encontrar la notación de [, ] para denotar la suma de morfismos y $\langle$, para el producto. En este documento se emplearán los símbolos de suma categórica en conjuntos $(+)$ y producto cartesiano usual $(\times)$ para denotar estas operaciones.
}

[Revista Integración, temas de matemáticas 


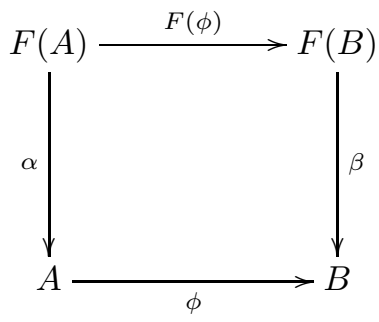

Más aún, no habrá lugar a confusión si nos referimos a $\phi$ como $F$-homomorfismo. Luego cada álgebra tipo 2,2,1,0 se puede pensar como un $\alpha: F(A) \rightarrow A$.

Este ejemplo es fácil de generalizar. Dada una signatura $\Sigma$ de símbolos de función con sus aridades finitas, considerando el funtor $F:$ Set $\rightarrow$ Set dado por $F(A)=\coprod_{f^{(n)} \in \Sigma} A^{n}$ observamos que guarda el tipo de $\Sigma$-álgebra; y cada $\Sigma$-álgebra $\mathbf{A}=\left\langle A, \Sigma^{\mathbf{A}}\right\rangle$ queda representada por $\alpha=\coprod_{f \in \Sigma} f^{\mathbf{A}}: \coprod_{f^{(n)} \in \Sigma} A^{n} \rightarrow A$. Para cada $f^{(n)} \in \Sigma$ tenemos que $f^{\mathbf{A}}=A^{n} \stackrel{i}{\longrightarrow} \coprod_{f(n) \in \Sigma} A^{n} \stackrel{\alpha}{\longrightarrow} A$, donde el primer morfismo es la inclusión apropiada de $A^{n}$ en $\coprod_{f^{(n)} \in \Sigma} A^{n}$. Así, cada $\Sigma$-álgebra $\mathbf{A}=\left\langle A, \Sigma^{\mathbf{A}}\right\rangle$ se representa por $\alpha: F(A) \rightarrow A$.

De otro lado, dadas dos $\Sigma$-álgebras $\mathbf{A}$ y $\mathbf{B}$, tenemos que una función $\phi: A \rightarrow B$ es un $\Sigma$ - homomorfismo si para toda $f^{(n)} \in \Sigma$ y para todos $a_{1}, \ldots, a_{n} \in A$ se cumple que $\phi\left(f^{\mathbf{A}}\left(a_{1}, \ldots, a_{n}\right)\right)=f^{\mathbf{B}}\left(\phi\left(a_{1}\right), \ldots, \phi\left(a_{n}\right)\right)$. Se define $F(\phi)=\coprod_{f(n) \in \Sigma} \prod^{n} \phi$. Así, tomando $\alpha=\coprod_{f \in \Sigma} f^{\mathbf{A}} \quad$ y $\beta=\coprod_{f \in \Sigma} f^{\mathbf{B}}$, el hecho de que $\phi$ sea un homomorfismo se reduce a que el siguiente diagrama conmuta:

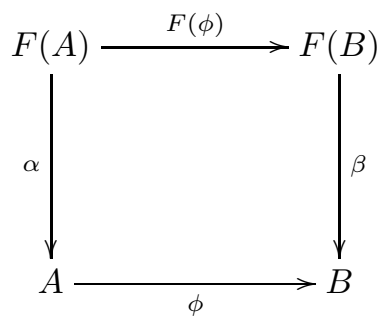

Por tanto tiene todo el sentido referirse a $\phi$ como un F-homomorfismo. Luego cada $\Sigma$-álgebra se puede pensar como un $\alpha: F(A) \rightarrow A$.

A partir de esta ilustración de las estructuras algebraicas del álgebra universal, vistas como álgebras asociadas a un funtor, esperamos cierta naturalidad en la siguiente generalización en la definición de álgebra.

Definición 2.2. Dado un funtor $F: C \rightarrow C$, una $F$-álgebra es un par $\langle A, \alpha\rangle$, donde $A$ es un objeto en $C$ y $\alpha: F(A) \longrightarrow A$. Un homomorfismo entre las álgebras $\langle A, \alpha\rangle$ y

Vol. 35, No. 1, 2017] 
$\langle B, \beta\rangle$ es un morfismo $h: A \rightarrow B$ para el cual el siguiente diagrama conmuta:

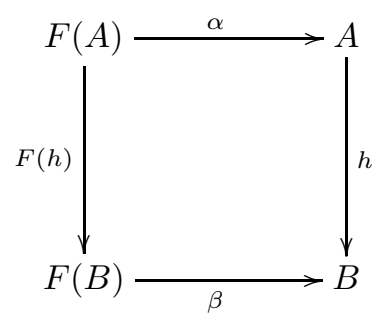

Las $F$-álgebras forman una categoría: $\mathbf{A l g}_{\mathbf{F}}$ para $F: C \rightarrow C$ fijo.

Tomemos como ejemplo el funtor $F:$ Set $\longrightarrow$ Set definido por $F(X)=X+1$. Si consideramos las funciones $s: \mathbb{N} \rightarrow \mathbb{N}$ y $o: 1 \rightarrow \mathbb{N}$, donde $1=\{\emptyset\}$, definidas por $s(n)=n+1$ y $o(\emptyset)=0$, es claro que $\langle\mathbb{N}, s+o: \mathbb{N}+1 \rightarrow \mathbb{N}\rangle$ es un elemento en $\mathbf{A} \lg _{\mathbf{F}}$. Veamos que $\langle\mathbb{N},(s, o)\rangle$ es inicial en $\mathbf{A} \lg _{\mathbf{F}}$. En efecto, para cualquier aplicación $f+x: X+1 \rightarrow X$, donde $x(\emptyset)=x_{0}$ con $x_{0} \in X$, considerando la aplicación $h: \mathbb{N} \rightarrow X$ definida por $h(0)=x_{0}$ y $h(s(n))=f(h(n))$ para todo $n \in \mathbb{N}$, tendremos que el siguiente diagrama conmuta:

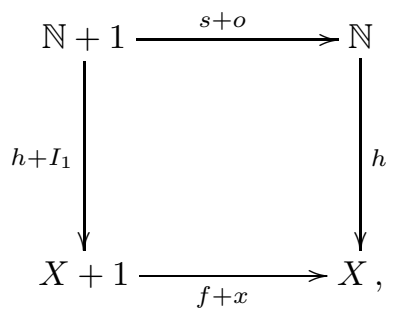

siendo $h$ un morfismo de $\mathbf{A l g}_{\mathbf{F}}$. La definición de $h$ garantiza su unicidad para cada $f$.

El hecho de ser $\mathbb{N}$ inicial en $\mathbf{A l g}_{\mathbf{F}}$ nos brinda el Principio de Inducción [7]; veamos: si tenemos $A \subseteq \mathbb{N}$ tal que $0 \in A$, y para todo $n \in A$ implica que $n+1 \in A$, mostremos que $A=\mathbb{N}$. Considerando $f+a: A+1 \rightarrow A$, donde $a(\emptyset)=0$ y $f(n)=n+1$, se tiene que $\langle A, f+a\rangle$ es un objeto de $\mathbf{A} \lg _{\mathbf{F}} ; \mathrm{y}$ como $\mathbb{N}$ es inicial en $\mathbf{A} \mathbf{l g}_{\mathbf{F}}$, existe un único homomorfismo $h$ para el que el siguiente diagrama conmuta:

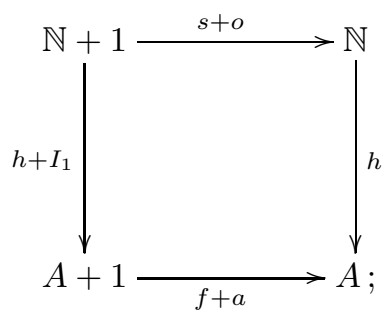

usando la inclusión $i: A \rightarrow \mathbb{N}$, tenemos el siguiente diagrama conmutativo:

[Revista Integración, temas de matemáticas 


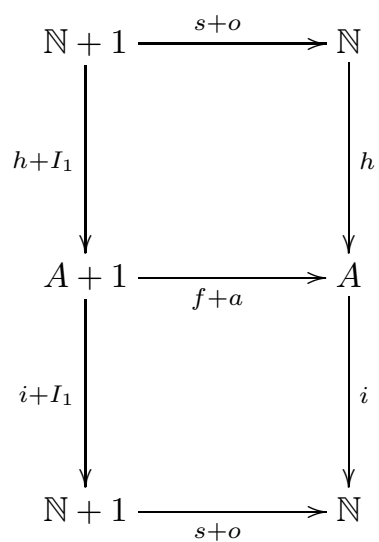

De donde, por ser $\mathbb{N}$ inicial, se obtiene que $i \circ h=I_{\mathbb{N}}$, y por lo tanto $A=\mathbb{N}$.

\section{Coálgebras y coinducción}

Se presenta una introducción a las coálgebras, concepto de singular importancia en ciencias de computación, pues como veremos captura en forma abstracta el concepto de autómata. El desarrollo de las coálgebras (conocimiento coinductivo) obedece a aportes de diversas áreas del conocimiento, entre otras los conjuntos no bien fundados, la teoría de sistemas y la lógica modal. Como ya lo habíamos anunciado, nuestra presentación está dentro del contexto de la teoría de categorías, y así formalizamos el concepto dual a $F$-álgebras. La relevancia se justifica en el desarrollo del presente trabajo, así como con nuestros comentarios finales en el mismo.

Recomendamos dos excelentes manuales para la aprehensión de coálgebra y coinducción; el primero de Pattinson [8], del que tomamos los ejemplos de Máquinas de Mealy y Modelos de Kripke, y el segundo de Jacobs-Rutten [6], del que hemos tomado el ejemplo de coálgebra final.

Definición 3.1. Para un funtor $F: C \rightarrow C$ una $F$-coálgebra es un par $\langle A, \alpha\rangle$, donde $A \in C$ y $\alpha: A \rightarrow F(A)$. Un homomorfismo entre las $F$-coálgebras $\langle A, \alpha\rangle$ y $\langle B, \beta\rangle$ es un morfismo $h: A \rightarrow B$ para el cual el siguiente diagrama conmuta:

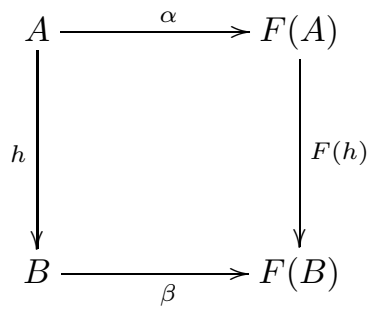

Las F-coálgebras forman una categoría: $\mathbf{C o a l g}$. El objeto final de $\mathbf{C o a l g}$, siempre que exista, se denomina coálgebra final para $F$.

Vol. 35, No. 1, 2017] 
El estudio de estos objetos finales es de gran relevancia. La existencia de la coálgebra final para $F$ guarda el comportamiento de cualquier $F$-coálgebra. Luego los funtores que admiten coálgebra final son de especial interés. En 1989 Aczel y Mendler mostraron que los funtores llamados $\omega$-accesibles o $\omega$-pequeños tienen coálgebra final. Dentro de estos están los denominados polinomiales obtenidos de la identidad, constantes, productos y potencias constantes. Una definición precisa de funtores polinomiales, junto al resultado antes mencionado y otros cercanos, se pueden ver en [8]. Los ejemplos que se consideran en el presente trabajo caen en su mayoría sobre esta última distinción. Además, si la categoría sobre la cual está definido el endofuntor $F$ tiene sumas amalgamadas, entonces por

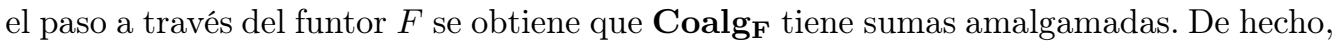
los endofuntores que consideraremos en el presente documento están definidos sobre la categoría Set, que tiene sumas amalgamadas, y por tanto nuestras categorías $\mathbf{C o a l g}$ F $_{\mathrm{F}}$ tienen sumas amalgamadas. La escogencia apropiada de los endofuntores garantiza mucha más estructura para las categorías de coálgebras.

\section{Ejemplo 3.2.}

\section{Máquinas de Mealy}

Una máquina de Mealy se puede considerar como una máquina que tiene un alfabeto de entrada $I$ y un alfabeto de salida $O$, en la que al ingresar un elemento del alfabeto de entrada, cambiará de estado y producirá un elemento del alfabeto de salida; formalmente, es una tripla $(S$, out, next) donde $S$ es el espacio de estados, next $: S \times I \rightarrow S$ y out $: S \times I \rightarrow O$.

Para ver a una máquina de Mealy como una coálgebra, es necesario que las funciones next y out tengan como dominio el espacio de estados $S$. Este cambio se puede hacer mediante la siguiente técnica, denominada currying:

Sea $f: A \times B \rightarrow C$ y $a \in A$ fijo. Obsérvese que la asignación $f(a,-): b \mapsto f(a, b)$ define una función de $B$ en $C$; con esto en mente, se puede definir una nueva función a partir de $f$ denominada curry, así: curry $(f): A \rightarrow C^{B}$ tal que $a \mapsto f_{a}$, donde $f_{a}: B \rightarrow C$ es la asignación mencionada arriba, es decir, la función que a cada $b$ le asigna el elemento $f(a, b)$.

Con lo anterior, tomando el producto cartesiano de las funciones out y next obtenemos

$$
\text { out } \times \text { next }: S \times I \rightarrow O \times S,
$$

con lo que

$$
\text { curry }(\text { out } \times \text { next }): S \rightarrow(O \times S)^{I},
$$

lo cual nos permite ver a las máquinas de Mealy como el par: $\langle S$, curry $($ out $\times$ next $)\rangle$, es decir, como una coálgebra sobre el funtor $F X=(O \times X)^{I}$.

\section{Modelos de Kripke}

Como en el caso anterior, un modelo de Kripke sobre un conjunto de proposiciones $A$ es una tripla $(S, R, V)$, donde $S$ es un espacio de estados o universos, $R \subseteq S \times S$ es una relación y $V: A \rightarrow \mathcal{P}(S)$ es una función denominada valuación. Dada $a \in A$, el modelo de Kripke funciona de la siguiente manera: la relación $R$ determina la evolución entre los universos, y la función valuación determina el conjunto de todos 
los universos en el que la proposición $a$ se satisface. Para que un modelo de Kripke sea una coálgebra, el objetivo es asociar a $S$ una función que tenga como dominio dicho conjunto; se puede ver la estructura de transición de un modelo de Kripke como una función next $: S \rightarrow \mathcal{P}(S)$. De la misma manera, a la función valuación se le puede asociar una función prop $: S \rightarrow \mathcal{P}(A)$ que a cada universo lo mapee en el conjunto de proposiciones que este satisface. Tomando el producto cartesiano de dichas funciones se obtendrá

$$
\text { next } \times \text { prop }: S \longrightarrow \mathcal{P}(S) \times \mathcal{P}(A) .
$$

Así, $\langle S$, next $\times$ prop $\rangle$ es una coálgebra sobre el funtor $F X=\mathcal{P}(X) \times \mathcal{P}(A)$.

\section{Un ejemplo de coálgebra final}

Dado un conjunto $A$, consideremos el endofuntor sobre la categoría de los conjuntos $F:$ Set $\longrightarrow$ Set definido por $F(X)=A \times X$. Tomando $A^{\omega}$ como el conjunto de todas las sucesiones infinitas de elementos de $A$, junto al morfismo head $\times$ tail : $A^{\omega} \rightarrow A \times A^{\omega}$ definido por head $(x)=x_{0}$ y $\operatorname{tail}(x)=\left(x_{1}, x_{2}, \ldots\right)$ tenemos que $\left\langle A^{\omega}\right.$, head $\times$ tail $\rangle$ es una $F$-coálgebra; más aún, es la coálgebra final para $F$. Veamos esta última afirmación: para cada $F$-coálgebra $\langle S, o \times t\rangle$, es decir, para cada $o \times t$ : $S \rightarrow A \times S$, definiendo $h$ por

$$
h(x)=\left(x_{0}, x_{1}, x_{2}, \ldots\right),
$$

donde $x_{i}=o \circ t^{i}(x)=o \circ \underbrace{t \circ t \circ t \circ \cdots \circ t}_{i-\text { veces }}$, se tiene que

$$
\operatorname{head}(h(x))=x_{0}=o(x)=1_{A}(o(x))
$$

y que

$$
\operatorname{tail}(h(x))=\left(x_{1}, x_{2}, \ldots\right)=\left(o \circ t(x), o \circ t^{2}(x), \ldots\right)=h(t(x)) .
$$

Es decir, el siguiente diagrama conmuta:

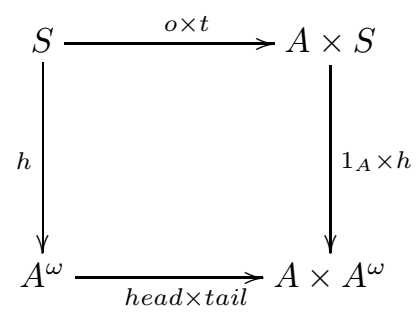

Además, para cada $F$-coálgebra $\langle S, o \times t\rangle$, de la misma definición $h$ resulta ser única.

Este ejemplo nos permite visualizar a $[0,1)$ como una coálgebra final. Basta considerar el conjunto $A=\{0,1,2, \cdots, 9\}$ y el intervalo $[0,1)$; todo $r \in[0,1)$ se puede escribir de la forma $r=0 . a_{1} a_{2} a_{3} \ldots$ con $a_{i} \in A$; así, aquí se encuentran todas las sucesiones infinitas de elementos de $A$, lo que permite concluir que $A^{\omega}$ se puede tomar como $[0,1)$ sin estructura.

Vol. 35, No. 1, 2017] 


\section{Cálculo coinductivo}

Las cadenas o sucesiones infinitas son objetos relativamente simples que proporcionan teorías muy valiosas, tanto en matemáticas como en ciencias de la computación. Las ecuaciones diferenciales en cadena $\mathrm{SDE}^{3}$ constituyen un método coinductivo para especificar cadenas y operaciones entre cadenas que se ha desarrollado en las últimas dos décadas en un sinnúmero de publicaciones. Este método permite desarrollar un cálculo en cadenas bastante similar al cálculo clásico del análisis matemático. Desarrollaremos aspectos básicos de este cálculo en la sección 3.4. Empezaremos en 3.1 con los autómatas de cadena; en 3.2 se estudia el comportamiento equivalente como bisimulación, una relación dual a las congruencias en álgebra universal; y en 3.3 veremos el comportamiento equivalente como una bisimulación, lo que nos permitirá dar el principio demostrativo de coinducción.

\subsection{Autómatas de cadena}

Un autómata de cadena se puede considerar como una máquina con una pantalla (que muestra un elemento $d$ de un conjunto de datos $A$ ) y un botón, en la que cada vez que se presione el botón, la pantalla se actualizará con un nuevo elemento $d^{\prime} \in A$ no necesariamente diferente al anterior. Un ejemplo bastante ilustrativo de estas son las clásicas máquinas tragamonedas en los casinos, que constan de una pantalla y un solo botón; sabemos que, al presionar el botón, la pantalla mostrará una combinación (no necesariamente diferente) de elementos. De esta manera, un autómata con un conjunto de estados internos $S$ (los datos) puede ser descrito mediante un par de funciones que determinan los elementos que pueden ser identificados y observados. Formalmente:

Definición 4.1. Sea $A$ un conjunto. Un autómata de cadena (o algunas veces simplemete autómata) sobre $A$ es una $F$-coálgebra $S=\langle S, o \times t\rangle$ consistente de un conjunto $S$ denominado espacio de estados y una función $o \times t: S \rightarrow A \times S$, donde $o: S \rightarrow A$ y $t: S \rightarrow S$ se denominan función de salida y transición, respectivamente.

Como vimos en la sección 2, las funciones head y tail juegan un papel importante en el cálculo de cadenas; recordemos la definición de estas funciones en términos que se adecúen al lenguaje que emplearemos. Recordemos que una cadena es una sucesión infinita de términos ordenados; formalmente, una cadena es una función $\sigma$ que a cada elemento del conjunto $\{0,1,2, \ldots\}$ le asigna un elemento de un conjunto $A$ y a cada una de estas asignaciones las ordena en una lista de la forma $(\sigma(0), \sigma(1), \sigma(2), \ldots)$. El conjunto de todas las cadenas con entradas en $A$ se denota por $A^{\omega}$ y se define de manera formal así:

$$
A^{\omega}=\{\sigma \mid \sigma:\{0,1,2, \ldots\} \longrightarrow A\} .
$$

Se define la función $O: A^{\omega} \rightarrow A$ mediante $O(\sigma)=\sigma(0)$. Al elemento $\sigma(0)$ se lo denomina el valor inicial de la cadena $\sigma$. También se define la derivada de la cadena $\sigma$ como la función $T: A^{\omega} \rightarrow A^{\omega}$ que a cada cadena $\sigma$ le asigna la cadena $T(\sigma)=\sigma^{\prime}=(\sigma(1), \sigma(2), \ldots)$.

Observación 4.2. Las funciones $O$ y $T$ corresponden a las funciones de salida y de transición (respectivamente) para el autómata cuyo espacio de estados es $A^{\omega}$.

\footnotetext{
${ }^{3}$ Abreviación de sus siglas en inglés: Stream Differential Equations.
}

[Revista Integración, temas de matemáticas 
En la siguiente sección se dará un sentido preciso a la siguiente definición.

Definición 4.3. Sea $\langle S, o \times t\rangle$ un autómata sobre $A$. La función $\llbracket-\rrbracket: S \rightarrow A^{\omega}$ definida por $\llbracket s \rrbracket=(o(s), o(t(s)), o(t(t(s))), \ldots)$ se denomina función de comportamiento.

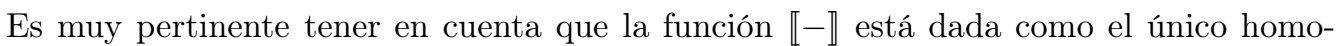
morfismo sobre la coálgebra final, con lo cual obtiene la siguiente proposición.

Proposición 4.4. La función de comportamiento es un homomorfismo.

\subsection{Comportamiento equivalente}

Para tener una idea intuitiva de lo que significa comportamiento equivalente considérese de nuevo que los autómatas son máquinas, y que ellas producen lo mismo mediante procesos que usted desconoce; sabiendo que los estados internos del sistema (en la máquina) son invisibles para el usuario, ¿cómo determinaría si los procesos son iguales? La respuesta a esta pregunta da lugar al concepto de comportamiento equivalente. El marco teórico presentado a continuación (hasta el final de la sección) será tomado de [8]. Para información detallada consultar esta referencia. Daremos las definiciones en forma general para coálgebras, pero cada vez estaremos volviendo sobre los autómatas de cadena.

Definición 4.5. Supóngase que $\langle C, \gamma\rangle,\langle D, \delta\rangle \in$ Coalg $_{F}$. Si $c \in C, d \in D$, se dice que son equivalentes en comportamiento $(c \approx d)$, si existen $\langle E, \epsilon\rangle \in \mathbf{C o a l g}_{F}$ y un par de homomorfismos $f: C \rightarrow E$ y $g: D \rightarrow E$ tales que $f(c)=g(d)$.

La prueba del ítem (iii) en la siguiente proposición requiere una construcción con sumas amalgamadas en la categoría Coalg $_{F}$, la cual está garantizada, pues nuestros endofuntores $F$ están definidos sobre la categoría Set, y por tanto Coalg $_{F}$ goza de esta cerradura.

Proposición 4.6. Sean $\langle C, \gamma\rangle,\langle D, \delta\rangle,\langle E, \epsilon\rangle \in \mathbf{C o a l g}_{F} y(c, d, e) \in C \times D \times E$. Entonces:

(i) $c \approx c$;

(ii) $c \approx d$ implica que $d \approx c$;

(iii) $c \approx d y d \approx e$ implica que $c \approx e$.

La restricción de la relación de comportamiento equivalente a una coálgebra $\langle C, \gamma\rangle$ se denota por $\approx_{C}$. Con lo cual tenemos el siguiente resultado.

Corolario 4.7. Sea $\langle C, \gamma\rangle \in \mathbf{C o a l g}_{F}$. Entonces $\approx_{C}$ es una relación de equivalencia.

Para una coálgebra fija, otra forma de establecer cuándo dos estados tienen comportamiento equivalente se ilustra mediante la siguiente proposición, que como técnica o principio demostrativo será mucho más eficiente que la definición.

Proposición 4.8. Sean $\langle C, \gamma\rangle \in \mathbf{C o a l g}_{F} y c, c^{\prime} \in C$. Las siguientes proposiciones son equivalentes:

(i) $c \approx c^{\prime}$;

Vol. 35, No. 1, 2017] 
(ii) Existen $\langle E, \epsilon\rangle \in \mathbf{C o a l g}_{F}$ y un morfismo $e:\langle C, \gamma\rangle \rightarrow\langle E, \epsilon\rangle$ que $e(c)=e\left(c^{\prime}\right)$.

El siguiente teorema permite establecer cuándo dos estados asociados a dos autómatas de cadena tienen un comportamiento equivalente. Para probarlo, es necesario tener primero el siguiente resultado.

Proposición 4.9. Si $\left\langle A_{1}, o_{1} \times t_{1}\right\rangle,\left\langle A_{2}, o_{2} \times t_{2}\right\rangle$ son autómatas y $f: A_{1} \rightarrow A_{2}$ es un homomorfismo entre ellos, entonces, para todo $a \in A_{1}$

$$
o_{1}\left(t_{1}^{n}(a)\right)=o_{2}\left(t_{2}^{n}(f(a))\right) .
$$

Demostración. Por ser $f$ un homomorfismo se cumple que

$$
o_{1}(a)=o_{2}(f(a)) \quad \mathrm{y} \quad f\left(t_{1}(a)\right)=t_{2}(f(a)) .
$$

Sea $n>0$; veamos que $f\left(t_{1}^{n}(a)\right)=t_{2}^{n}(f(a))$ :

$$
\begin{aligned}
f\left(t_{1}^{n}(a)\right) & =f\left(t_{1}\left(t_{1}^{n-1}(a)\right)\right) \\
& =t_{2}\left(f\left(t_{1}^{n-1}(a)\right)\right) \\
& =t_{2}\left(f\left(t_{1}\left(t_{1}^{n-2}(a)\right)\right)\right) \\
& =t_{2}^{2}\left(f\left(t_{1}^{n-2}(a)\right)\right) \\
& \vdots \\
& =t_{2}^{n}(f(a)) .
\end{aligned}
$$

Así, tenemos que $o_{2}\left(t_{2}^{n}(f(a))\right)=o_{2}\left(f\left(t_{1}^{n}(a)\right)\right)=o_{1}\left(t_{1}^{n}(a)\right)$.

Teorema 4.10. Sean $\left\langle A_{1}, o_{1} \times t_{1}\right\rangle,\left\langle A_{2}, o_{2} \times t_{2}\right\rangle$ autómatas $y\left(a, a^{\prime}\right) \in A_{1} \times A_{2}$; entonces $a \approx a^{\prime}$ si y solo si $\llbracket a \rrbracket=\llbracket a^{\prime} \rrbracket$.

Demostración. Supongamos que $a \approx a^{\prime}$; entonces existen un autómata $\left(A_{3},\left\langle o_{3}, t_{3}\right\rangle\right)$ y homomorfismos $f: A_{1} \rightarrow A_{3}$ y $g: A_{2} \rightarrow A_{3}$ tales que $f(a)=g\left(a^{\prime}\right)$. Como $f$ y $g$ son homomorfismos, de la proposición anterior tenemos que para todo $n$,

$$
o_{1}\left(t_{1}^{n}(a)\right)=o_{2}\left(t_{2}^{n}(f(a))\right)=o_{2}\left(t_{2}^{n}\left(g\left(a^{\prime}\right)\right)\right)=o_{1}\left(t_{1}^{n}\left(a^{\prime}\right)\right) .
$$

Así,

$\left(o_{1}(a), o_{1}\left(t_{1}(a)\right), o_{1}\left(t_{1}^{2}(a)\right), \ldots, o_{1}\left(t_{1}^{n}(a)\right), \ldots\right)=\left(o_{1}\left(a^{\prime}\right), o_{1}\left(t_{1}\left(a^{\prime}\right)\right), o_{1}\left(t_{1}^{2}\left(a^{\prime}\right)\right), \ldots, o_{1}\left(t_{1}^{n}\left(a^{\prime}\right)\right), \ldots\right)$,

es decir, $\llbracket a \rrbracket=\llbracket a^{\prime} \rrbracket$. Recíprocamente, supongamos que $\llbracket a \rrbracket=\llbracket a^{\prime} \rrbracket ;$ como consecuencia de la proposición 4.4 se tiene que $a \approx a^{\prime}$.

\subsection{Comportamiento equivalente como bisimulación}

En esta parte es necesario detenernos brevemente en dos clases de coálgrebras: simples y extensivas. La importancia de los sistemas simples radica en que estos permiten hacer demostraciones empleando coinducción; dicho de una mejor manera, satisfacen el siguiente principio de demostración, que va a representar un papel indispensable en la demostración de algunos de los resultados principales, como el teorema fundamental del cálculo y la unicidad de la solución de ecuaciones diferenciales. Mientras que las extensivas incorporan todos los comportamientos observables.

[Revista Integración, temas de matemáticas 
Definición 4.11. Sea $\langle C, \gamma\rangle \in \mathbf{C o a l g}_{F}$. Decimos que $\langle C, \gamma\rangle$ es:

- Simple, si $c=c^{\prime}$ siempre que $c \approx c^{\prime}$.

- Extensiva, si para todas $\langle D, \delta\rangle$ y $d \in D$ existe $c \in C$ tal que $c \approx d$.

Es decir, en una coálgebra simple tener dos estados con comportamiento equivalente implica que son iguales, y que las coálgebras extensivas realizan todos los comportamientos observables. El teorema 2.7.2 de [8], que enunciamos a continuación, establece la equivalencia entre las coálgebras finales, y las simples y extensivas.

Teorema 4.12. Sea $\langle C, \gamma\rangle \in \mathbf{C o a l g}_{F}$. Las siguientes proposiciones son equivalentes:

(i) $\langle C, \gamma\rangle$ es una coálgebra final;

(ii) $\langle C, \gamma\rangle$ es simple y extensiva.

Para finalizar esta sección introduciremos de manera general el concepto de bisimulación y su relación con la equivalencia de comportamiento; particularmente, ilustraremos cómo se evidencia esta en los autómatas de cadena.

Definición 4.13. Sean $\langle C, \gamma\rangle$ y $\langle D, \delta\rangle F$-coálgebras. Una relación $B \subseteq C \times D$ es una bisimulación si existe $\beta: B \rightarrow F B$ tal que las proyecciones $\pi_{1}: B \rightarrow C$ y $\pi_{2}: B \rightarrow D$ sean homomorfismos de coálgebras, es decir, si el siguiente diagrama conmuta:

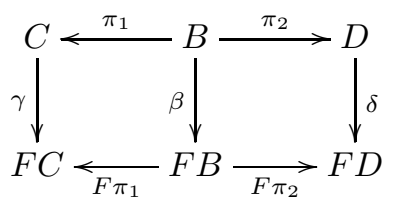

Diremos que $c \in C$ y $d \in D$ son bisimilares, y lo denotaremos por $c \sim d$, si existe una bisimulación $B$ para la cual $(c, d) \in B$.

El autor en [8] logra hacer una conexión entre los conceptos de bisimulación y comportamiento equivalente mediante la siguiente proposición:

Proposición 4.14. Sean $\langle C, \gamma\rangle,\langle D, \delta\rangle \in \operatorname{Coalg}_{F}$ y $c \in C, d \in D$. Si $c \sim d$, entonces $c \approx d$.

Y como consecuencia inmediata de esta y el Teorema 4.12, tenemos el siguiente corolario:

Corolario 4.15. Sea $\langle C, \gamma\rangle \in \mathbf{C o a l g}_{F}$ simple y extensiva. Si $c, c^{\prime} \in C$ y $c \sim c^{\prime}$, entonces $c=c^{\prime}$.

Como hemos insistido recurrentemente, nos interesan los autómatas de cadena. Es muy importante conocer explícitamente su coálgebra final (si la tiene), así que en lo que queda de esta sección ilustraremos brevemente los resultados obtenidos arriba para autómatas de cadena en pro de ello.

Vol. 35, No. 1, 2017] 
Sabemos que el endofuntor en la categoría Set asociado a los autómatas de cadena está dado por $F(X)=A \times X$, siendo $A$ el conjunto de salida (o de etiquetas). Consideremos los autómatas de cadena $\left\langle C, o_{1} \times t_{1}\right\rangle$ y $\left\langle D, o_{2} \times t_{2}\right\rangle$. Si $R \subseteq C \times D$ es una bisimulación, se tiene entonces que el siguiente diagrama conmuta:

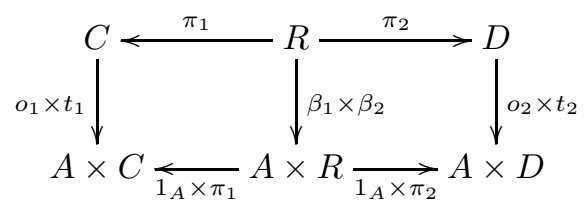

Así, $o_{1}\left(\pi_{1}(c, d)\right)=\beta_{1}(c, d)=o_{2}\left(\pi_{2}(c, d)\right)$, lo que implica que $o_{1}(c)=o_{2}(d), \forall(c, d) \in R$.

Por otro lado, como el dominio y el codominio de $\beta_{2}$ es $R, \mathrm{y} R \subseteq C \times D$, entonces, tomando $\beta_{2}((c, d))=(a, e)$, tenemos que

$$
t_{1}(c)=\pi_{1}((a, e))=a \quad y \quad t_{2}(d)=\pi_{2}((a, e))=e .
$$

De donde $\left(t_{1}(c), t_{2}(d)\right) \in R$ para todo $(c, d) \in R$.

Si reemplazamos los espacios de estados $C$ y $D$ por $A^{\omega}$, lo anterior lo podemos resumir en la siguiente definición:

Definición 4.16. Una bisimulación en $A^{\omega}$ es una relación $R \subseteq A^{\omega} \times A^{\omega}$ tal que para cualesquiera $\sigma$ y $\tau$ en $A^{\omega}$, se tiene

$$
\sigma R \tau \Rightarrow\left\{\begin{array}{rrr}
\sigma(0) & = & \tau(0) \\
\sigma^{\prime} & R & \tau^{\prime}
\end{array}\right.
$$

Es fácil probar que la unión de todas las bisimulaciones es una bisimulación (de hecho, la más grande); a esta se la denomina relación de bisimilaridad (que corresponde a la definición de atrás).

La importancia del siguiente teorema radica en que de él se derivan los resultados principales de esta sección: la finalidad de $\left\langle A^{\omega}, O \times T\right\rangle$ en $\mathbf{C o a l g}_{F}$ y el teorema de coinducción.

Teorema 4.17. $\left\langle A^{\omega}, O \times T\right\rangle$ es simple.

Demostración. Sean $\sigma, \sigma^{\prime} \in A^{\omega}$. Si $\sigma \approx \sigma^{\prime}$, entonces por el Teorema 4.10 tenemos que $\llbracket \sigma \rrbracket=\llbracket \sigma^{\prime} \rrbracket ;$ esto quiere decir que

$$
\left(O(\sigma), O(T(\sigma)), \ldots, O\left(T^{n}(\sigma)\right), \ldots\right)=\left(O\left(\sigma^{\prime}\right), O\left(T\left(\sigma^{\prime}\right)\right), \ldots, O\left(T^{n}\left(\sigma^{\prime}\right)\right), \ldots\right),
$$

de donde

$$
O\left(T^{n}(\sigma)\right)=O\left(T^{n}\left(\sigma^{\prime}\right)\right) \quad \forall n \geq 0
$$

lo que implica que

$$
\sigma(n)=\sigma^{\prime}(n) \quad \forall n \geq 0,
$$

pues el valor inicial de cada derivada corresponde a la primera componente de esta, con lo que la cadena formada por todos estos valores iniciales corresponde a la cadena en sí. Por lo tanto, $\sigma=\sigma^{\prime}$. 
Como los homomorfismos entre coálgebras preservan los comportamientos observables, tenemos que si para toda $\langle D, \delta\rangle$ en $\mathbf{C o a l g}_{F}$ existe un homomorfismo $f:\langle D, \delta\rangle \rightarrow\langle C, \gamma\rangle$, entonces $\langle C, \gamma\rangle$ es extensiva. Este es el caso de $\left\langle A^{\omega}, O \times T\right\rangle$ por la Proposición 3.1, y así tenemos que $\langle O \times T\rangle$ es extensiva. Luego, como una consecuencia del teorema anterior y del Teorema 4.12, tenemos el siguiente corolario:

Corolario 4.18. $\left\langle A^{\omega}, O \times T\right\rangle$ es una coálgebra final.

Como una consecuencia del teorema anterior y el Corolario 4.15 tenemos el siguiente corolario:

Corolario 4.19. (Coinducción) Para todo $\sigma, \tau \in A^{\omega}, \sigma \sim \tau$ implica que $\sigma=\tau$.

\section{Serie de Taylor}

Considere el conjunto

$$
\mathscr{A}=\{f: \mathbb{R} \longrightarrow \mathbb{R} \mid f \text { es analítica en } 0\} .
$$

Sabemos que una función al ser analítica en un punto lo será en una vecindad alrededor de él, y por lo tanto será infinitamente diferenciable en dicha vecindad. Con esto presente, podemos definir las siguientes funciones:

$$
\begin{array}{cc}
o: \mathscr{A} \rightarrow \mathbb{R} & t: \mathscr{A} \rightarrow \mathscr{A} \\
o(f)=f(0), & t(f)=f^{\prime},
\end{array}
$$

donde $f^{\prime}$ corresponde a la derivada de la función $f$. Esto quiere decir que $\mathscr{A}$ se puede ver como un autómata, siendo o y $t$ las funciones de salida y transición asociadas a $\mathscr{A}$. Así, la función de comportamiento tendrá la forma

$$
\llbracket f \rrbracket=\left(f(0), f^{\prime}(0), f^{\prime \prime}(0), \ldots\right) \quad \forall f \in \mathscr{A},
$$

es decir, el único homomorfismo de $\mathscr{A}$ en $\mathbb{R}^{\omega}$ está definido mediante la expresión de arriba, que es la serie de Taylor de $f$ y se denota por:

$$
\mathscr{T}(f)=\left(f(0), f^{\prime}(0), f^{\prime \prime}(0), \ldots\right) .
$$

\subsection{Cálculo básico de cadenas}

Ahora, como habíamos anunciado desde la introducción de esta sección, presentaremos aspectos básicos del método coinductivo SDE para especificar cadenas y operaciones entre cadenas. Con lo cual haremos un acercamiento al cálculo en cadenas que nos permita ilustrar el carácter coinductivo del cálculo clásico del análisis matemático real. A fin de disponer de una buena estructura algebraica en las cadenas, se parte de cadenas sobre un conjunto con una adecuada estructura algebraica. En principio, si consideramos las cadenas sobre $\langle R,+, \cdot, 0,1\rangle$ un anillo, es bien conocido que estas cadenas $R^{\omega}$ gozan de una estructura de anillo con las operaciones definidas para cualesquiera $a, b \in R^{\omega}$ por $(a+b)(n)=a(n)+b(n)$ y $(a \times b)(n)=\Sigma_{k+l=n} a(k) b(l)$, el módulo aditivo $0(n)=0$ para todo $n \in \omega ;$ y el modulo multiplicativo $1(0)=1$ y $1(n)=0$ para todo $n>0$. Constituyen

Vol. 35, No. 1, 2017] 
las series de potencias formales sobre el anillo $R$, usualmente denotadas por $R[[X]]$, que resulta ser conmutativo siempre que $R$ lo sea. Además se tiene la inmersión del anillo $R$ en $R[[X]]$, identificando cada $r \in R$ por la cadena $[r]=(r, 0,0,0,0, \ldots)$. Usando SDE $[r]$ es la única cadena que satisface la ecuación diferencial $[r]^{\prime}=[0]$ y $[r](0)=r$. Igualmente, usando SDE, las operaciones se pueden definir para $\sigma$ y $\tau$ cadenas por

- La suma de $\sigma$ y $\tau$ como la única cadena que satisface la ecuación diferencial

$$
(\sigma+\tau)^{\prime}=\sigma^{\prime}+\tau^{\prime} \quad y \quad(\sigma+\tau)(0)=\sigma(0)+\tau(0) .
$$

- El producto ${ }^{4} \sigma \times \tau$ como la única cadena que satisface ecuación diferencial

$$
(\sigma \times \tau)^{\prime}=\left(\sigma^{\prime} \times \tau\right)+\left([\sigma(0)] \times \tau^{\prime}\right) \quad y \quad(\sigma \times \tau)(0)=\sigma(0) \tau(0)
$$

Es decir, tenemos que $\left\langle R^{\omega},+, \times\right\rangle$ es un anillo, el cual es conmutativo siempre que $R$ lo sea, con módulo aditivo [0] y unidad [1]. De otro lado, por la definición del producto se obtiene que una cadena $\sigma$ es invertible si y sólo si $\sigma(0)$ es una unidad en el anillo $R$. En particular, cuando $R$ es un cuerpo tenemos que $\sigma$ es invertible si y sólo si $\sigma(0) \neq 0$.

Además en este cálculo se tiene la cadena constante $X=(0,1,0,0,0, \ldots)$, que es la única cadena que satisface la ecuación diferencial $X^{\prime}=[1]$ y $X(0)=0$. Esta cadena tiene la propiedad de que para cada cadena $\sigma, \mathbf{X} \times \sigma=\sigma \times \mathbf{X}=(0, \sigma(0), \sigma(1), \sigma(2), \ldots)$. Luego la multiplicación por $X$ representa una suerte de "integración de cadena" vista como el inverso a la derivación de cadena. Lo que nos permite dar el teorema fundamental del cálculo en cadenas tomado de Rutten [10].

Teorema 4.20. Sea $\sigma \in R^{\omega}$. Entonces, $\sigma=[\sigma(0)]+\mathbf{X} \times \sigma^{\prime}$.

Demostración. Sea $B=\left\{\langle\sigma, \sigma\rangle: \sigma \in R^{\omega}\right\} \cup\left\{\left\langle\sigma,[\sigma(0)]+\mathbf{X} \times \sigma^{\prime}\right\rangle: \sigma \in R^{\omega}\right\}$. Veamos que $B$ es una bisimulación:

$$
\begin{aligned}
\left([\sigma(0)]+\mathbf{X} \times \sigma^{\prime}\right)(0) & =[\sigma(0)](0)+\left(\mathbf{X} \times \sigma^{\prime}\right)(0) \\
& =\sigma(0)+\mathbf{X}(0) \sigma^{\prime}(0) \\
& =\sigma(0)+0 \sigma^{\prime}(0) \\
& =\sigma(0)+0=\sigma(0)
\end{aligned}
$$

Por otro lado,

$$
\begin{aligned}
\left([\sigma(0)]+\mathbf{X} \times \sigma^{\prime}\right)^{\prime} & =[\sigma(0)]^{\prime}+\left(\mathbf{X} \times \sigma^{\prime}\right)^{\prime} \\
& =[0]+\mathbf{X}^{\prime} \times \sigma^{\prime}+[\mathbf{X}(0)] \times \sigma^{\prime \prime} \\
& =[0]+\left([1] \times \sigma^{\prime}\right)+\left([0] \times \sigma^{\prime \prime}\right) \\
& =\sigma^{\prime} .
\end{aligned}
$$

Así, tenemos de $B$ que $\sigma^{\prime} B\left(\sigma^{\prime}=\left([\sigma(0)]+\mathbf{X} \times \sigma^{\prime}\right)^{\prime}\right)$, de donde se concluye que $B$ es una bisimulación y por lo tanto, por coinducción, se tiene la igualdad deseada.

\footnotetext{
${ }^{4}$ Es usual en la literatura de ciencias de la computación referirse a este producto como producto convolución.
}

[Revista Integración, temas de matemáticas 
Ahora veamos una deducción de lo establecido en el teorema anterior para un tipo especial de cadenas: las series de Taylor.

Sea $f \in \mathscr{A}$ (ver ejemplo de la Serie de Taylor de la sección anterior). Para $f$, el teorema fundamental del cálculo asegura que

$$
f(x)=f(0)+\int_{0}^{x} f^{\prime}(t) d t .
$$

Supóngase que $\sigma=\mathscr{T}(f)$. Veamos que para este tipo de cadenas se puede deducir lo establecido en el teorema anterior a partir de su definición:

$$
\begin{aligned}
\sigma & =\mathscr{T}(f(x)) \\
& =\mathscr{T}\left(f(0)+\int_{0}^{x} f^{\prime}(t) d t\right) .
\end{aligned}
$$

Pero $\mathscr{T}(f(0))=(f(0), 0,0, \ldots)$ y $\mathscr{T}\left(\int_{0}^{x} f^{\prime}(t) d t\right)=\left(0, f^{\prime}(0), f^{\prime \prime}(0), \ldots\right)$. Así pues,

$\sigma=\mathscr{T}(f(0))+\mathscr{T}\left(\int_{0}^{x} f^{\prime}(t) d t\right)$.

Note además que $\mathscr{T}(f(0))=[\mathscr{T}(f)(0)]$, es decir,

$\sigma=[\mathscr{T}(f)(0)]+\mathscr{T}\left(\int_{0}^{x} f^{\prime}(t) d t\right)$.

Ahora, veamos que la relación

$$
R=\left\{\langle\sigma, \sigma\rangle \mid \sigma \in \mathbb{R}^{\omega}\right\} \cup\left\{\left\langle\mathscr{T}\left(\int_{0}^{x} f(t) d t\right), \mathbf{X} \times \mathscr{T}(f(t))\right\rangle \mid f \in \mathscr{A}\right\}
$$

es una bisimulación:

Sean $\sigma=\mathscr{T}\left(\int_{0}^{x} f(t) d t\right)$ y $\tau=\mathrm{X} \times \mathscr{T}(f(t))$ para $f \in \mathscr{A}$. Tenemos que $\sigma(0)=0=\tau(0)$. Por otro lado, es facil probar que $\sigma^{\prime}=\tau^{\prime}$, y de $R$ se sabe que $\tau^{\prime} R \tau^{\prime}$, lo que quiere decir que $\sigma^{\prime} R \tau^{\prime}$, lo que nos lleva a que $R$ es una bisimulación, y por el corolario de coinducción 4.19 , podemos concluir que

$$
\mathscr{T}\left(\int_{0}^{x} f(t) d t\right)=\mathbf{X} \times \mathscr{T}(f(t)) .
$$

Por lo tanto, continuando en $(*)$

$$
\begin{aligned}
\sigma & =[\mathscr{T}(f)(0)]+\mathbf{X} \times \mathscr{T}\left(f^{\prime}(t) d t\right) \\
& =[\sigma(0)]+\mathbf{X} \times \sigma^{\prime} .
\end{aligned}
$$

\section{Sistemas de ecuaciones diferenciales}

A fin de ilustrar la fortaleza de SDE necesitamos formalizar los sistemas de ecuaciones en el marco coinductivo. Empezaremos a manera de introducción con los sistemas de ecuaciones simples, para pasar a los lineales, que es nuestro propósito en este documento. La existencia y unicidad de las soluciones de tales sistemas está determinada por la coinducción. Logros y desarrollos más generales, así como ciertos detalles técnicos no incluidos en el presente, se puede consultar en [5].

Vol. 35, No. 1, 2017] 


\subsection{Sistemas de ecuaciones diferenciales simples}

Consideremos la ecuación

$$
\sigma^{\prime}=\sigma \quad \text { y } \quad \sigma(0)=r \in R
$$

La solución es una cadena que tenga como valor inicial el elemento $r$ y que al derivarla obtengamos la misma cadena. A simple vista, se puede ver que la solución es $\sigma=(r, r, r, \ldots)$. Daremos uso a las herramientas teóricas para buscar dicha solución y mostrar su unicidad: Sea $S=(\{s\}, o \times t)$ un autómata con los operadores definidos así: $o(s)=r$ y $t(s)=s$. Sean $\sigma \in R^{\omega}$ y $h: S \longrightarrow R^{\omega}$ un morfismo tal que $h(s)=\sigma$. Por la finalidad de $R^{\omega}$ tenemos que $h$ es un homomorfismo, y por lo tanto que el siguiente diagrama conmuta

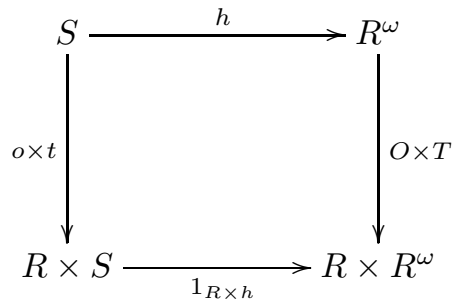

Así, tenemos que

$$
\begin{aligned}
\sigma^{\prime} & =T(\sigma) \\
& =T(h(s)) \text { por definición de } h \\
& =h(t(s)) \text { por conmutatividad del diagrama } \\
& =h(s)=\sigma .
\end{aligned}
$$

De la misma manera, $\sigma(0)=O(\sigma)=O(h(s))=o(s)=r$. Lo que nos lleva a concluir que $\sigma$ es la cadena que satisface (1). Para garantizar la unicidad de ella, supongamos que existe $\rho \in R^{\omega}$ tal que $\rho^{\prime}=\rho$ y $\rho(0)=r$. Veamos que $\{(\rho, \sigma)\}$ es una bisimulación. Tenemos que $\rho(0)=r=\sigma(0)$; además, $\rho R \sigma$ y se tiene que $\rho^{\prime}=\rho$ y $\sigma^{\prime}=\sigma$, de donde se concluye que $\rho^{\prime} R \sigma^{\prime}$; luego, $\rho \sim \sigma$ y por el corolario de coinducción $\rho=\sigma$.

Este ejemplo está dentro de las ecuaciones más simples. Tomaremos ahora la formalización de [10] para los sistemas de este estilo.

Fijemos $R$ sólo como un conjunto, llamaremos un sistema de ecuaciones simple sobre las variables $X=\left\{x_{i} \mid i \in I\right\}$ a un conjunto simultáneo de SDEs de la forma

$$
x^{\prime}=y_{i} \quad y \quad x_{i}(0)=r_{i},
$$

donde $r_{i} \in R$ y $y_{i} \in X$ para todo $i \in I$. Nuestro interés está sólo para cuando $X$ es finito. Un sistema simple está plenamente determinado por una función $e: X \rightarrow R \times X$, es decir, por un autómata de cadena con espacios de estado $X$. Una solución de $e: X \rightarrow R \times X$ es una aplicación $h: X \rightarrow R^{w}$ que preserve las ecuaciones $h\left(x_{i}\right)(0)=r_{i}$ y $h\left(x_{i}\right)^{\prime}=h\left(y_{i}\right)$ para todo $i \in I$. Lo cual corresponde a que el siguiente diagrama conmuta:

[Revista Integración, temas de matemáticas 


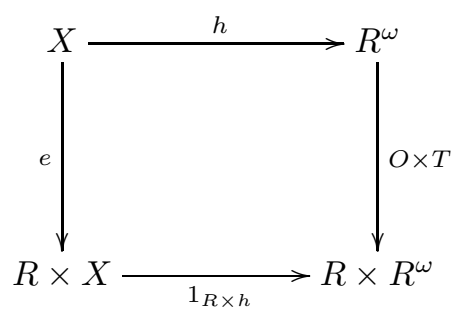

La solución del sistema es el único homomorfismo de cadena de $\langle X, e\rangle$ al autómata de cadena final. Usando el principio de coinducción, tenemos que todo sistema de ecuaciones simples siempre tiene solución y es única. Diremos que $\sigma \in R^{w}$ es una solución de $e$ si existe $x \in X$ tal que $\sigma=h(x)$, y en tal caso también diremos que es una especificación de $\sigma$. Las soluciones de sistemas de ecuaciones simples finitos son exactamente los comportamientos de autómatas de cadena finitos, los cuales son precisamente los eventualmente periódicos.

\subsection{Sistemas de ecuaciones diferenciales lineales}

En lo que resta de este escrito, centrado en [5], $R$ se fija como un cuerpo numérico, con lo cual $R^{\omega}$ tiene estructura de espacio vectorial sobre $R$. Se define la suma y el producto escalar puntualmente. Además dado un conjunto $X$ denotaremos por $\mathcal{V}(X)$ al espacio libre generado por $X$. Es decir, el conjunto de todas las combinaciones lineales formales sobre $X$;

$$
\mathcal{V}(X)=\left\{r_{1} x_{1}+r_{2} x_{2}+\cdots+r_{n} x_{n} \mid r_{i} \in R, x_{i} \in X, 1 \leq i \leq n\right\}
$$

Un sistema de ecuaciones lineales sobre las variables $X=\left\{x_{i} \mid i \in I\right\}$ es un conjunto simultáneo de SDEs de la forma

$$
x^{\prime}=y_{i} \quad y \quad x_{i}(0)=r_{i},
$$

donde $r_{i} \in R$ y $y_{i} \in \mathcal{V}(X)$ para todo $i \in I$. Nuestro interés está sólo para cuando $X$ es finito. Es decir, un sistema lineal es una función $e=\langle o, d\rangle: X \rightarrow R \times \mathcal{V}(X)$. Una solución de $e$ es una aplicación $h: X \rightarrow R^{\omega}$ que preserve las ecuaciones $h\left(x_{i}\right)(0)=o\left(x_{i}\right)$, y si $d\left(x_{i}\right)=r_{1} \cdot x_{1}+r_{2} \cdot x_{2}+\cdots+r_{n} \cdot x_{n}$, entonces $h\left(x_{i}\right)^{\prime}=r_{1} \cdot h\left(x_{1}\right)+r_{2} \cdot h\left(x_{2}\right)+\cdots+r_{n} \cdot h\left(x_{n}\right)$ para todo $i \in I$. Para ver las soluciones de los sistemas de ecuaciones diferenciales lineales es indispensable verlos como autómatas sobre espacios vectoriales.

Un autómata de cadena lineal es un autómata sobre espacios vectoriales, es decir, es una aplicación de la forma $\langle o, d\rangle: V \rightarrow R \times V$, donde $V$ es un espacio vectorial sobre $R, \mathrm{y}$ $o$ y $d$ son funciones lineales. Un homomorfismo de autómatas de cadena lineales es una aplicación entre los espacios vectoriales de estados, la cual es homomorfismo de autómatas de cadena y es lineal. Como $\mathcal{V}(X)$ es el espacio libre generado por $X$, dado un sistema de ecuaciones lineales $e: X \rightarrow R \times \mathcal{V}(X)$ existe una única extensión lineal (un autómata de cadena lineal) $e^{*}: \mathcal{V}(X) \rightarrow R \times \mathcal{V}(X)$; además, el autómata de cadena final es también un autómata de cadena lineal final. Se demuestra por coinducción que todo sistema de

Vol. 35, No. 1, 2017] 
ecuaciones lineales tiene una única solución. Cuando $X$ es finito el único homomorfismo lineal $h: \mathcal{V}(X) \rightarrow R^{\omega}$ puede representarse por una matriz finita con cadenas racionales como entradas. Una cadena $\sigma \in R^{\omega}$ es racional si es de la forma

$$
\sigma=\frac{a_{0}+\left(a_{1} \times \mathbf{X}\right)+\left(a_{2} \times \mathbf{X}^{2}\right)+\cdots+\left(a_{n} \times \mathbf{X}^{n}\right)}{b_{0}+\left(b_{1} \times \mathbf{X}\right)+\left(b_{2} \times \mathbf{X}^{2}\right)+\cdots+\left(b_{m} \times \mathbf{X}^{m}\right)}
$$

donde $a_{i}, b_{j} \in R$ con $b_{0} \neq 0$ y $n, m \in \mathbb{N}$ con $1 \leq i \leq n$ y $1 \leq j \leq m$.

Recordemos que si $R$ es un anillo, entonces el conjunto $M_{n}(R)$ de matrices $n \times n$ con entradas en $R$ con la suma y la multiplicación usual de matrices también es un anillo. Más aún, si $R$ es conmutativo, podemos definir la multiplicación por escalar en $M_{n}(R)$ como sigue: para todo $r \in R$ y toda matriz $M \in M_{n}(R), r \cdot M=\left(r M_{i j}\right)_{i j}$, y se tiene la propiedad $r \cdot(M N)=(r \cdot M) N=M(r \cdot N)$, con lo cual $M_{n}(R)$ es una $R$-álgebra asociativa. Ahora, volviendo a nuestra restricción de que $R$ es un cuerpo, entonces tenemos que $M_{n}\left(R^{\omega}\right)$ es una $R^{\omega}$-álgebra asociativa.

Ahora nos concentraremos en el caso $n=2$, es decir, en los sistemas de ecuaciones lineales en dos variables. Ilustramos el método de solución matricial en este caso, que es fácilmente generalizable a $n$ variables. Un sistema de ecuaciones lineales en dos variables,

$$
\begin{array}{ll}
\sigma_{1}^{\prime}=m_{11} \cdot \sigma_{1}+m_{12} \cdot \sigma_{2} & \sigma_{1}(0)=r_{1} \\
\sigma_{2}^{\prime}=m_{21} \cdot \sigma_{1}+m_{22} \cdot \sigma_{2} & \sigma_{2}(0)=r_{2}
\end{array}
$$

puede ser representado en forma matricial mediante:

$$
\left(\begin{array}{l}
\sigma_{1} \\
\sigma_{2}
\end{array}\right)^{\prime}=\left(\begin{array}{ll}
m_{11} & m_{12} \\
m_{21} & m_{22}
\end{array}\right)\left(\begin{array}{l}
\sigma_{1} \\
\sigma_{2}
\end{array}\right) \quad \text { y } \quad\left(\begin{array}{l}
\sigma_{1} \\
\sigma_{2}
\end{array}\right)(0)=\left(\begin{array}{l}
r_{1} \\
r_{2}
\end{array}\right)=Y_{0}
$$

con $m_{i j} \in R$ vistos como $\left[m_{i j}\right] \in R^{\omega}$ donde $i, j=1,2$.

Empleando el teorema fundamental del cálculo, se llega a que la solución para el sistema está dada por:

$$
\left(\begin{array}{l}
\sigma_{1} \\
\sigma_{2}
\end{array}\right)=\left[I_{2}-\mathrm{X} \cdot M\right]^{-1} Y_{0}
$$

Para finalizar, se desarrollarán ejemplos en los que se pretende ilustrar la técnica de solución matricial desarrollada atrás, pues uno de los objetivos de este documento es mostrar diferentes técnicas empleadas en métodos coinductivos y coalgebraicos. Estos ejemplos ya han sido desarrollados sin manipulación de matrices [9].

\section{Solución a un sistema de dos ecuaciones lineales}

Considere el sistema

$$
\begin{array}{ll}
\sigma^{\prime}=\tau & \sigma(0)=1 \\
\tau^{\prime}=\sigma & \tau(0)=0
\end{array}
$$

[Revista Integración, temas de matemáticas 
En este caso, la solución es $\sigma=(1,0,1,0, \ldots)$, Veamos

De acuerdo con la ecuación (2), la solución al sistema está dada por

$$
\left(\begin{array}{l}
\sigma \\
\tau
\end{array}\right)=\frac{1}{1-\mathrm{X}^{2}} \cdot\left(\begin{array}{cc}
1 & \mathrm{X} \\
\mathrm{X} & 1
\end{array}\right)\left(\begin{array}{l}
1 \\
0
\end{array}\right)
$$

de donde obtenemos que $\sigma$ y $\tau$ corresponden a las cadenas racionales

$$
\sigma=\frac{1}{1-\mathrm{X}^{2}} \quad \tau=\frac{\mathrm{X}}{1-\mathrm{X}^{2}} .
$$

Luego, computando los valores inciales tenemos que

$$
\sigma(0)=\frac{1}{1-\mathbf{X}^{2}}(0)=1 \quad y \quad \tau(0)=\frac{\mathbf{X}}{1-\mathbf{X}^{2}}(0)=0
$$

Nótese que basta con estos valores iniciales para reconstruirlas completamente, pues cada una es la derivada de la otra.

\section{Cadena de Fibonacci}

Considere la ecuación con valores iniciales

$$
\sigma^{\prime \prime}=\sigma^{\prime}+\sigma \quad\left\{\begin{array}{c}
\sigma(0)=0 \\
\sigma^{\prime}(0)=1
\end{array}\right.
$$

Sabemos que toda ecuación diferencial de orden $n \geq 2$ se puede escribir como un sistema de $n$ ecuaciones diferenciales de primer orden; llevando a cabo el mismo proceso en este caso, obtendremos para la ecuación de segundo grado un sistema de dos ecuaciones de primer orden. Es decir, haciendo la sustitución $\tau=\sigma^{\prime}$ obtenemos, a partir del sistema inicial, el siguiente sistema de ecuaciones de primer orden:

$$
\begin{array}{ll}
\tau=\sigma^{\prime} & \sigma(0)=0 \\
\tau^{\prime}=\tau+\sigma & \tau(0)=1 .
\end{array}
$$

De la misma manera que en el ejemplo anterior, la representación matricial del sistema está dada por

$$
\left(\begin{array}{l}
\sigma \\
\tau
\end{array}\right)^{\prime}=\left(\begin{array}{ll}
0 & 1 \\
1 & 1
\end{array}\right)\left(\begin{array}{l}
\sigma \\
\tau
\end{array}\right) \quad y \quad\left(\begin{array}{l}
\sigma \\
\tau
\end{array}\right)(0)=\left(\begin{array}{l}
0 \\
1
\end{array}\right) .
$$

Así, la solución dada mediante la ecuación 2 se ve representada por:

$$
\begin{aligned}
& \left(\begin{array}{l}
\sigma \\
\tau
\end{array}\right)=\left(\begin{array}{cc}
1 & -\mathrm{X} \\
-\mathrm{X} & 1-\mathrm{X}
\end{array}\right)^{-1}\left(\begin{array}{l}
0 \\
1
\end{array}\right) \\
& =\left(\begin{array}{cc}
\frac{1-X}{1-X-X^{2}} & \frac{X}{1-X-X^{2}} \\
\frac{X}{1-X-X^{2}} & \frac{1}{1-X-X^{2}}
\end{array}\right)\left(\begin{array}{l}
1 \\
0
\end{array}\right) \\
& =\left(\frac{\frac{X}{1-X-X^{2}}}{\frac{1}{1-X-X^{2}}}\right) \text {, }
\end{aligned}
$$

Vol. 35, No. 1, 2017] 
de donde se obtiene que

$$
\sigma=\frac{\mathrm{X}}{1-\mathrm{X}-\mathrm{X}^{2}}
$$

cuya expansión en serie está dada por

$$
X+X^{2}+2 X^{3}+3 X^{4}+5 X^{5}+\cdots,
$$

que tiene como coeficientes los números de Fibonacci.

Otra manera de obtener sigma es computando sucesivamente los valores iniciales de las derivadas de la forma recional de $\sigma$. Haciendo esto, se obtiene:

$$
\begin{aligned}
\sigma(0) & =\frac{X(0)}{[1](0)-X(0)-X^{2}(0)}=0, \\
\sigma^{\prime}(0) & =\frac{[1](0)}{[1](0)-X(0)-X^{2}(0)}=1, \\
\sigma^{\prime \prime}(0) & =\sigma(0)+\sigma^{\prime}(0)=1, \\
& \vdots \\
\sigma^{n+1}(0) & =\sigma^{n}(0)+\sigma^{n-1}(0)=F_{n}+F_{n-1},
\end{aligned}
$$

formando la cadena cuyas entradas (a excepción del 0) son los números de la sucesión de Fibonnacci.

\section{A manera de conclusión}

Queremos resaltar que gracias a técnicas relativamente elementales se visualizan como coálgebras ciertos autómatas y semánticas de la lógica modal. Las Máquinas de Mealy a través de la técnica denominada currying, y los Modelos de Kripke utilizando el funtor partes para ver "funciones" multivaluadas como verdaderas funciones. El uso de matrices permite ver varias cadenas simultáneas en una sola, lo que proporciona la solución de sistemas ecuaciones diferenciales lineales. Por último la bisimulación como técnica demostrativa, que en nuestro caso pone de presente el carácter coinductivo del análisis real. Las especificaciones de SDE presentadas en este documento se pueden extender a lenguajes libres de contexto [5].

Además, existen presentaciones coinductivas de otras áreas relevantes de las matemáticas, entre las cuales destacamos las de Aczel [1], Fauser-Pavlovic [3] y Gumm [4]. Aczel presenta modelos no bien fundados para las Teoría de Conjuntos; Gumm utiliza el endofuntor filtro $\mathcal{F}:$ Set $\rightarrow$ Set, definido sobre los objetos por $\mathcal{F}(X)$ como la colección de todos los filtros sobre $X$, para capturar cada espacio topológico $\langle A, \tau\rangle$ como la $\mathcal{F}$-coálgebra $\alpha: A \rightarrow \mathcal{F}(A)$ definida; para todo $a \in A$, por $\alpha(a)$ igual a la colección de todas las vecindades de $a$; y Fauser-Pavlovic en el marco de las variedades y haces tangentes proporcionan una semántica de los funtores haz tangente y haz cotangente, y dan un tratamiento coalgebraico a la Segunda Ley de Newton que les permite ir más allá de la mecánica clásica, y adentrarse en la teoría de la relatividad.

[Revista Integración, temas de matemáticas 


\section{Referencias}

[1] Aczel P., Non-Well-Founded Sets, Stanford University, Center for the Study of Language and Information, 1988.

[2] Burris S. and Sankappanavar H.P., A Course in Universal Algebra, Springer-Verlarg, New York, 1981.

[3] Fauser B. and Pavlovic D., "Smooth coalgebra: testing vector analysis", Math. Structures Comput. Sci. (2015), 1-41.

[4] Gumm P., "Functor for Coalgebras", Algebra Universalis 45 (2001), No. 2-3, 135-147.

[5] Hansen H.H., Kupke C. and Rutten J.J., "Stream differential equations: specifications formats and solution methods", Log. Methods Comput. Sci 13 (2017), No. 1, 1-51.

[6] Jacobs B. and Rutten J.J., "A tutorial on (Co)algebras and (Co)induction", EATCS Bulletin 62 (1997), 222-259.

[7] Ortiz G. and Valencia S., "La categoricidad de los reales en Hilbert", Rev. Bras. Hist. Mat. 10 (2010), No. 19, 39-65.

[8] Pattinson D., An introduction to the Theory of Coalgebras, Course notes Institut für Informatik, München, 2003.

[9] Rutten J.J., "A coinductive calculus of streams", Math. Structures Comput. Sci. 15 (2005), No. $193-147$.

[10] Rutten J.J., "Behavioural differential equations: a coinductive calculus of streams, automatas and power series", Theoret. Comput. Sci. 308 (2003), 1-53.

Vol. 35, No. 1, 2017] 